\title{
TRANSLATION OF RELATION SCHEMES AND SOME RELATED PROBLEMS
}

\author{
NGUYEN HOANG SON \\ Department of Mathematics, College of Sciences, Hue University
}

\begin{abstract}
The keys play important roles in the relational databases design theory. The results of keys have been widely investigated, they can be seen in $[4,5,6]$. In [4] to find minimal keys of relation scheme $S=(U, F)$, we translate relation scheme $S$ to relation scheme $\widetilde{S}$, which has a less number of attributes and shorter functional dependencies. in this translation relation scheme, finding minimal keys becomes much more simple. The aim of this article is to investigate some properties of the translation relation scheme $\widetilde{S}$ and some related problems.

Tóm tăt. Khóa đóng vai trò quan trọng trong lý thuyết thiết kế cơ sờ dữ liệu quan hệ. Các kết quả về khóa đã được nghiên cứu khá nhiều, có thể tìm thấy các kết quà này trong $[4,5,6]$. Trong [4] đề tìm khoá tối tiểu của sơ đồ quan hệ $S=(U, F)$, chúng ta chuyền dịch sơ dồ quan hệ $S$ về sơ đồ quan hệ $\widetilde{S}$, là sơ đồ có ít thuộc tính hơn và các phụ thuộc hàm ngắn gọn hơn. Trong sơ đồ quan hệ chuyển dịch này việc tìm khóa tối tiểu trờ nên đơn giản hơn. Mục đích của bài báo này là nghiên cứu một số tính chất của sơ đồ quan hệ chuyển dịch $\widetilde{S}$ và một số vấn đề liên quan.
\end{abstract}

\section{INTRODUCTION}

Let us give some necessary definitions and results that are used in the next section. The concepts are given in this section can be found in $[1,2,3,5]$.

Definition 1.1. Let $U=\left\{a_{1}, \ldots, a_{n}\right\}$ be a nonempty finite set of attributes. A functional dependency (FD) is a statement of form $X \rightarrow Y$, where $X, Y \subseteq U$. The FD $X \rightarrow Y$ holds in a relation $R=\left\{h_{1}, \ldots, h_{m}\right\}$ over $U$ if

$$
\left(\forall h_{i}, h_{j} \in R\right)\left((\forall a \in X)\left(h_{i}(a)=h_{j}(a)\right) \Rightarrow(\forall b \in Y)\left(h_{i}(b)=h_{j}(b)\right)\right) .
$$

We also say that $R$ satisfies the FD $X \rightarrow Y$.

Let $F_{R}$ be a family of all FDs that holds in $R$.

Definition 1.2. Then $F=F_{R}$ satisfies

(F1) $X \rightarrow X \in F$,

(F2) $(X \rightarrow Y \in F, Y \rightarrow Z \in F) \Rightarrow(X \rightarrow Z \in F)$,

(F3) $(X \rightarrow Y \in F, X \subseteq V, W \subseteq Y) \Rightarrow(V \rightarrow W \in F)$,

(F4) $(X \rightarrow Y \in F, V \rightarrow W \in F) \Rightarrow(X \cup V \rightarrow Y \cup W \in F)$.

A family of FDs satisfying (F1) - (F4) is called an $f$-family over $U$. 
Clearly, $F_{R}$ is an $f$-family over $U$. It is known [1] that if $F$ is an arbitrary $f$-family, then there is a relation $R$ over $U$ such that $F_{R}=F$.

Given a family $F$ of FDs over $U$, there exists a unique minimal $f$-family $F^{+}$that contains $F$. It can be seen that $F^{+}$contains all FDs which can be derived from $F$ by the rules (F1)(F4).

A relation scheme $\mathrm{S}$ is a pair $(U, F)$, where $U$ is a set of attributes, and $F$ is a set of FDs over $U$.

Denote $X^{+}=\left\{a \in U: X \rightarrow\{a\} \in F^{+}\right\} . X^{+}$is called the closure of $X$ over $S$.

It is clear that $X \rightarrow Y \in F^{+}$iff $Y \subseteq X^{+}$.

Definition 1.3. Let $S=(U, F)$ be a relation scheme over $U, K \subseteq U$. $K$ is called a minimal key of $S$, if it satisfies the following two conditions:

(1) $K \rightarrow U \in F^{+}$,

(2) $\nexists K^{\prime} \subset K$ such that $K^{\prime} \rightarrow U \in F^{+}$.

The subset $K$ which satisfies only (1) is called a key of $S$.

\section{RESULTS}

Let $S=(U, F)$ be a relation scheme, where $U=\left\{a_{1}, a_{2}, \ldots, a_{n}\right\}$ is a set of attributes, and $F=\left\{L_{i} \rightarrow R_{i}: L_{i}, R_{i} \subseteq U, L_{i} \cap R_{i}=\emptyset, i=1,2, \ldots, m\right\}$ is a set of FDs over $U$.

Denote

$$
L=\bigcup_{i=1}^{m} L_{i}, R=\bigcup_{i=1}^{m} R_{i} .
$$

The following theorem is known [6].

Theorem 2.1. ([6]) Let $S=(U, F)$ be a relation scheme over $U$ and $K$ be a minimal key of $S$. Then

$$
(U-R) \subseteq K \subseteq(U-R) \cup((L \cap R)-a(L, R)),
$$

where $a(L, R)=(L \cap R) \cap(L-R)^{+}$.

Definition 2.2. Let $S=(U, F)$ be a relation scheme over $U$. Set $\widetilde{U}=(L \cap R)-(L-R)^{+}$, and $\widetilde{F}=\left\{L_{i} \cap \widetilde{U} \rightarrow R_{i} \cap \widetilde{U}: L_{i} \cap \widetilde{U} \neq \emptyset, R_{i} \cap \widetilde{U} \neq \emptyset, L_{i} \rightarrow R_{i} \in F\right\}$. Then $\widetilde{S}=(\widetilde{U}, \widetilde{F})$ is called a translation relation scheme of $S$ over $\widetilde{U}$.

In [4] we proved the following result.

Theorem 2.3. Let $S=(U, F)$ be a relation scheme over $U, \widetilde{S}=(\widetilde{U}, \widetilde{F})$ is a translation relation scheme of $S$ over $\widetilde{U}$, and $K \subseteq \widetilde{U}$. Then, $K$ is a minimal key of $\widetilde{S}$ if and only if $K \cup(U-R)$ is a minimal key of $S$.

Denote $K_{S}$ the set of all minimal keys of $S$. From Theorem 2.3 we obtain the following corollaries.

Corollary 2.4. If $K \in K_{\widetilde{S}}$, then there exists $K^{\prime} \in K_{S}$ such that $K \subseteq K^{\prime}$.

Corollary 2.5. If $U-R=\emptyset$ then $K_{\widetilde{S}}=K_{S}$.

The following corollary is also clear. 
Corollary 2.6. Let $S=(U, F)$ be a relation scheme, where $U=\left\{K_{1}, K_{2}, \ldots, K_{m}\right\}$ and $F=\left\{K_{1} \rightarrow U, K_{2} \rightarrow U, \ldots, K_{m} \rightarrow U\right\}$. Then, $\widetilde{U}=U, \widetilde{F}=F$ and hence $K_{\widetilde{S}}=K_{S}$.

Remark 1. For every $L_{i}^{\prime} \rightarrow R_{i}^{\prime} \in \widetilde{F},\left(L_{i}^{\prime}\right)_{\widetilde{F}}^{+}=\widetilde{U}$ is not hold, i.e. $L_{i}^{\prime}$ is not the key of $\widetilde{S}$ and so it is not the minimal key. For example, we consider $F=\{\{a, b\} \rightarrow\{c\},\{d\} \rightarrow\{a\},\{c\} \rightarrow\{b, d\}\}$ over $U=\{a, b, c, d\}$. Then, we have $L=\{a, b, c, d\}, R=\{a, b, c, d\}, L \cap R=\{a, b, c, d\}, L-$ $R=\emptyset$, and hence $\widetilde{U}=\{a, b, c, d\}, \widetilde{F}=\{\{a, b\} \rightarrow\{c\},\{d\} \underset{\widetilde{U}}{\longrightarrow}\{a\},\{c\} \rightarrow\{b, d\}\}$. It is obvious that, with a FD $\{d\} \rightarrow\{a\} \in \widetilde{F}$ we have $\{d\}_{\widetilde{F}}^{+}=\{a, d\} \neq \widetilde{U}$.

In translation relation schemes $\widetilde{S}=(\widetilde{U}, \widetilde{F})$, FDs and attributes have some rather interesting properties as follows.

Theorem 2.7. Let $\widetilde{S}=(\widetilde{U}, \widetilde{F})$ be a translation relation scheme of $S=(U, F)$ trên $\widetilde{U}$. Then

(i) If $a \in \widetilde{U}$, then there exists $L_{i}^{\prime} \rightarrow R_{i}^{\prime} \in \widetilde{F}$ such that $a \in R_{i}^{\prime}$.

(ii) If $a \in \widetilde{U}$, then there exists $L_{j}^{\prime} \rightarrow R_{j}^{\prime} \in \widetilde{F}$ such that $a \in L_{j}^{\prime}$.

Proof. (i) Since $a \in \widetilde{U}$, it is obvious that $a \in L \cap R$. Thus there exists a FD $L_{i} \rightarrow R_{i} \in F$ such that $a \in R_{i}$. Therefore we have $a \in R_{i} \cap \widetilde{U}$, i.e. $R_{i} \cap \widetilde{U} \neq \emptyset$. Furthermore, $L_{i} \cap \widetilde{U} \neq \emptyset$. In fact, if $L_{i} \cap \widetilde{U}=\emptyset$, then

$$
L_{i} \subseteq(L-R)^{+},
$$

or

$$
L-R \rightarrow L_{i} \in F^{+} .
$$

On the other hand, we have

$$
L_{i} \rightarrow R_{i} \in F, R_{i} \rightarrow\{a\} \in F^{+} .
$$

From (1) and (2) we have $L-R \rightarrow\{a\} \in F^{+}$, i.e. $a \in(L-R)^{+}$, which contradicts the hypothesis $a \in \widetilde{U}$. Hence $L_{i} \cap \widetilde{U} \neq \emptyset$. Set $L_{i}^{\prime}=L_{i} \cap \widetilde{U}, R_{i}^{\prime}=R_{i} \cap \widetilde{U}$ we have (i), i.e. there exists a FD $L_{i}^{\prime} \rightarrow R_{i}^{\prime} \in \widetilde{F}$ such that $a \in R_{i}^{\prime}$.

(ii) Because $a \in \widetilde{U}$, we have $a \in L \cap R$, i.e. there exists a FD $L_{j} \rightarrow R_{j} \in F$ such that $a \in L_{j}$. Therefore

$$
a \in L_{j} \cap \widetilde{U} \text {. }
$$

Moreover, we have

$$
\left(L_{j}\right)_{F}^{+} \cap \widetilde{U} \subseteq\left(L_{j} \cap \widetilde{U}\right)_{\widetilde{F}}^{+} .
$$

In fact, according to the algorithm for finding the closure $L_{j}^{+}$of $L_{j}$ with $\left(L_{j}\right)_{F}^{(0)}=L_{j},\left(L_{j} \cap\right.$ $\widetilde{U})_{\widetilde{F}}^{(0)}=L_{j} \cap \widetilde{U}$, we have

$$
\left(L_{j}\right)_{F}^{(0)} \cap \widetilde{U} \subseteq\left(L_{j} \cap \widetilde{U}\right)_{\widetilde{F}}^{(0)}
$$

is trivial. Assume that

$$
\left(L_{j}\right)_{F}^{(k)} \cap \widetilde{U} \subseteq\left(L_{j} \cap \widetilde{U}\right)_{\widetilde{F}}^{(k)} .
$$

Then

$$
\begin{aligned}
\left(L_{j}\right)_{F}^{(k+1)} \cap \widetilde{U} & =\left(\left(L_{j}\right)_{F}^{(k)} \cup\left\{b: L_{i} \rightarrow R_{i} \in F, b \in R_{i}, L_{i} \subseteq\left(L_{j}\right)_{F}^{(k)}\right\}\right) \cap \widetilde{U} \\
& =\left(\left(L_{j}\right)_{F}^{(k)} \cap \widetilde{U}\right) \cup\left(\left\{b: L_{i} \rightarrow R_{i} \in F, b \in R_{i}, L_{i} \subseteq\left(L_{j}\right)_{F}^{(k)}\right\} \cap \widetilde{U}\right) \\
& \subseteq\left(L_{j} \cap \widetilde{U}\right)_{\widetilde{F}}^{(k)} \cup\left(\left\{b: L_{i} \rightarrow R_{i} \in F, b \in R_{i}, L_{i} \subseteq\left(L_{j}\right)_{F}^{(k)}\right\} \cap \widetilde{U}\right) .
\end{aligned}
$$


On the other hand, from assumption (4) and $L_{i} \subseteq\left(L_{j}\right)_{F}^{(k)}$ we have

So

$$
L_{i} \cap \widetilde{U} \subseteq\left(L_{j}\right)_{F}^{(k)} \cap \widetilde{U} \subseteq\left(L_{j} \cap \widetilde{U}\right)_{F}^{(k)} .
$$

$$
\begin{aligned}
\left(L_{j}\right)_{F}^{(k+1)} \cap \widetilde{U} & \subseteq\left(L_{j} \cap \widetilde{U}\right)_{\widetilde{F}}^{(k)} \cup\left(\left\{b: L_{i} \rightarrow R_{i} \in F, b \in R_{i}, L_{i} \subseteq\left(L_{j}\right)_{F}^{(k)}\right\} \cap \widetilde{U}\right) \\
& \subseteq\left(L_{j} \cap \widetilde{U}\right)_{\widetilde{F}}^{(k+1)} .
\end{aligned}
$$

Hence, (3) has been proved, i.e.

$$
\left(L_{j}\right)_{F}^{+} \cap \widetilde{U} \subseteq\left(L_{j} \cap \widetilde{U}\right)_{\widetilde{F}}^{+} .
$$

Moreover $L_{j} \rightarrow R_{j} \in F$, thus $R_{j} \subseteq\left(L_{j}\right)_{F}^{+}$. Consequently

$$
R_{j} \cap \widetilde{U} \subseteq\left(L_{j}\right)_{F}^{+} \cap \widetilde{U} \subseteq\left(L_{j} \cap \widetilde{U}\right)_{\widetilde{F}}^{+} .
$$

It shows that

$$
L_{j} \cap \widetilde{U} \rightarrow R_{j} \cap \widetilde{U} \in \widetilde{F} .
$$

Set $L_{j}^{\prime}=L_{j} \cap \widetilde{U}, R_{j}^{\prime}=R_{j} \cap \widetilde{U}$, we have (ii), i.e. there exists a FD $L_{j}^{\prime} \rightarrow R_{j}^{\prime} \in \widetilde{F}$ such that $a \in L_{j}^{\prime}$.

The theorem is proved.

From Theorem 2.7, we have the following corollaries.

Corollary 2.8. For each $L_{i}^{\prime} \rightarrow R_{i}^{\prime} \in \widetilde{F}$, if $a \in R_{i}^{\prime}$ then $a \in L_{j}^{\prime}$, where $L_{j}^{\prime} \rightarrow R_{j}^{\prime} \in \widetilde{F}$.

Corollary 2.9. For each $L_{i}^{\prime} \rightarrow R_{i}^{\prime} \in \widetilde{F}$, if $a \in L_{i}^{\prime}$ then $a \in R_{j}^{\prime}$, where $L_{j}^{\prime} \rightarrow R_{j}^{\prime} \in \widetilde{F}$.

Theorem 2.10. Let $S=(\widetilde{U}, F)$ be a relation scheme over $U$ and $\widetilde{S}=(\widetilde{U}, \widetilde{F})$ be a translation relation scheme of $S$ over $\widetilde{U}$. Then

(i) If $L_{i} \rightarrow R_{i} \in F$ such that $L_{i} \cap \widetilde{U}=\emptyset$, then

$$
\forall a \in \widetilde{U}: a \notin R_{i} \text { and hence } L_{i} \cap \widetilde{U} \rightarrow R_{i} \cap \widetilde{U} \notin \widetilde{F} \text {. }
$$

(ii) If $L_{i} \rightarrow R_{i} \in F$ such that $R_{i} \cap \widetilde{U}=\emptyset$, then

$$
\forall a \in \widetilde{U}: a \notin L_{i} \text { and hence } L_{i} \cap \widetilde{U} \rightarrow R_{i} \cap \widetilde{U} \notin \widetilde{F} \text {. }
$$

Proof. (i) Since $L_{i} \cap \widetilde{U}=\emptyset$, we have $L_{i} \subseteq(L-R)^{+}$. Thus

$$
L-R \rightarrow L_{i} \in F^{+} .
$$

Assume $a \in R_{i}$, it implies that $R_{i} \rightarrow\{a\} \in F^{+}$. On the other hand, we have $L_{i} \rightarrow R_{i} \in F$. Hence, by (F2) in the Definition 1.2 we have

$$
L-R \rightarrow\{a\} \in F^{+},
$$

or

$$
a \in(L-R)^{+},
$$


which contradicts the hypothesis $a \in \widetilde{U}$. Thus $a \notin R_{i}$, and $R_{i} \cap \widetilde{U}=\emptyset$, i.e.

$$
L_{i} \cap \widetilde{U} \rightarrow R_{i} \cap \widetilde{U} \notin \widetilde{F} .
$$

(ii) Suppose $a \in L_{i}$, which implies that $a \in L_{i} \cap \widetilde{U}$. With the similar provement like Theorem 2.7 , we also obtain

$$
L_{i} \cap \tilde{U} \rightarrow R_{i} \cap \tilde{U} \in \widetilde{F}
$$

i.e.

$$
R_{i} \cap \tilde{U} \neq \emptyset
$$

which contradicts the hypothesis $R_{i} \cap \widetilde{U}=\emptyset$. So $a \notin L_{i}$, and hence we have

$$
L_{i} \cap \tilde{U} \rightarrow R_{i} \cap \tilde{U} \notin \widetilde{F} .
$$

The theorem is proved.

Note that, if an attribute $a \in U$ appears only in either the left side or the right side or none of the FDs in $F$, then $a$ will not be in $\widetilde{U}$, i.e., if $a \in L-R$ or $a \in R-L$ or $a \notin L \cup R$ then $a \notin \widetilde{U}$.

\section{REFERENCES}

[1] Armstrong W.W., Dependency Structure of Database Relationship, Information Processing 74, North-Holland Pub. Co., 1974, 580-583.

[2] Codd E. F., A relational model for large shared data banks, Comm. ACM 13 (1970) $337-387$.

[3] Demetrovics J., Thuan H. , Bao L. V., and Huy N. X., Translation of relation schemes, Balanced relation schemes and the problem of key representation, J. Inf. Process. Cybern. EIK 23 (1987) 81-97.

[4] Son N. H., Hung N. V., Some result s about keys of relation schemes, Journal of Computer Science and Cybernetics 18 (2002) 285-289.

[5] Thi V.D., Minimal keys and Antikeys, Acta Cybernetica 7 (1986) 361-371.

[6] Thuan H., Bao L. V., Some results about key of relational schemas, Acta Cybernetica (1985) 99-113.

Received on August 10, 2004 Revised on December 13, 2004 